\title{
Aspectos metodológicos de la historia de la comunicación
}

\author{
Dra. Mercedes Román Portas \\ Profesora Titular de Periodismo \\ Universidad de Vigo
}

\section{RESUMEN}

En este artículo se propone el estatuto científico de la comunicación como punto de partida para una metodología de la historia de la comunicación. Se hace referencia al objeto de la comunicación e invita a centrarse en los resultados de esta ciencia para conocer cuestiones del pasado que orienten en la búsqueda de soluciones a problemas similares de la actualidad. Además, en la conformación de las mentalidades contemporáneas, juegan un papel clave los medios de comunicación social, por tanto la historia de las mentalidades parece un buen anclaje metodológico para los historiadores de la comunicación.

\begin{abstract}
ABSTRAC
This article intends the scientific statute of communication like an starting point for a methodology of communication history. The article is focus in the results of this science to know questions of the past that guide in the search of solutions to similar problems in present time. Also, in the conformation of contemporary mentalities, social media play a key paper, therefore the history of the mentalities seems a good methodological anchorage for the historians of the communication.
\end{abstract}

Palabras claves: Comunicación/Historia de la comunicación/Metodología/Mentalidad/Actualidad.

Key Words: Communication/Communication History/Methodology/Mentality/Present Time.

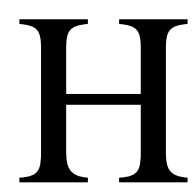
abitualmente, las reflexiones sobre la historia de la comunicación se inician con una amplia referencia a lo que se podría considerar, además con rigor, otra historia. Es decir, a la narración de un proceso, en el que el protagonismo corresponde a una ciencia -la historia- que en su desarrollo interno se va abriendo a campos diversos. Uno de ellos sería la comunicación social. El inconveniente mayor de este enfoque es, que sitúa la historia de la comunicación, como un mero apéndice de la historia general. Esta tendencia había ganado fuerza, a medida que la propia ciencia histórica conoció una revolución metodológica que pretendía conseguir una historia total. 
Cabría pensar que la definición del concepto de historia de la comunicación, exigiría previamente la de la propia historia. No se puede negar el sentido y el valor de tal empeño metodológico pero en este artículo se pretende ofrecer un planteamiento diverso.

La concepción de la metodología de la historia de la comunicación que propongo, toma como punto de partida el estatuto científico de la comunicación. Ahí me parece que es, donde debe ponerse el acento. Luego vendrá la tarea de situar en ese plano general, la importancia de los procesos es decir, la perspectiva de la diacronía y la concreción de la cronología. Es claro que ese empeño intelectual por lo histórico, no es un añadido superfluo: como no lo es en ninguna ciencia social.

Comenzaré por intentar fijar con la máxima claridad posible, el objeto de la comunicación, ya que es la materia sobre cuyo pasado, se va a reflexionar.

La comunicación como objeto científico es una acción significativa que tiene como finalidad que otro comprenda lo que significa esa acción y que sea efectivamente comprendida. Al considerar el objeto como una acción, la ciencia de la comunicación se integra automáticamente en el ámbito de la Ciencia General de la Acción Social ${ }^{1}$.

Esta consideración ofrece dos ventajas. Primero, define el objeto de estudio de la ciencia de la comunicación. Luego, da un ámbito apropiado a la historia de la comunicación.

Voy a tratar a continuación de las características esenciales del fenómeno comunicativo. Tomaré como punto de partida que la comunicación entra dentro del ámbito de las acciones humanas. Por eso tiene estas cinco características esenciales: es humana, es social, es referencial, es compleja y se da en presente interior o vivido como señala Martín Algarra en su artículo "Sobre la complejidad terminológica de la teoría de la comunicación" ${ }^{2}$. Detallaré brevemente una por una:

1) La comunicación es humana. Es un fenómeno exclusivamente humano. Es decir libre, capaz de acción significativa.

2) La comunicación es siempre social; para que se dé comunicación hacen falta, al menos, dos personas. Eso quiere decir que siempre está referida a otro, que pretende afectar a alguien distinto del yo que comunica.

1 "Una premisa importante: el estudio formal de la comunicación puede ser caracterizado con exactitud como una ciencia social» (FISHER, B. A.: Perspectives on Human Communication. Nueva York 1978, p. 20). Apreciaciones cercanas en NÚÑEZ LADEVEZE, Luis: La Construcción de Texto. Madrid 1991, pp. 12 y ss.

2 Cfr. MARTÍN ALGARRA, Manuel. "Sobre la complejidad terminológica de la teoría de la comunicación" en Estudios de Periodismo, 2, 1993, pp.7-26. 
3) La comunicación es referencial. Es un peculiar modo de compartir, sin pérdida por parte de nadie. Se puede decir que al comunicar se comparte el mundo de un modo espiritual, puesto que todas las realidades, físicas o no, pueden tenerse en la mente en forma de conocimientos. Al fin y al cabo cuando comunicamos algo a alguien queremos que ese alguien conozca ese algo que nosotros conocemos, aunque él no haya podido contemplar directamente la realidad que hemos expresado. Lo que se comparte es conocimiento. Y el conocimiento es siempre conocimiento de algo. Esto implica que existe una realidad, un mundo común objetivo, dotado de entidad metafísica y cognoscible. Pero también implica que ese mundo común lo percibimos todos de un modo más o menos parecido, y que la comunicación se realiza por medio de un elemento físico, el código que se comparte.

4) La comunicación es una realidad compleja. Se trata de una realidad transaccional. Es un proceso complejo en el cual la gente maneja mensajes y crea significados. Esta complejidad reafirma el ya mencionado carácter social de la comunicación: es un proceso que exige, al menos, dos sujetos empíricos.

Parece evidente, que esta característica, introduce la intencionalidad como un elemento importante. Me refiero a la intencionalidad comunicativa, y como fruto de ella, podemos referirnos a la eficacia comunicativa.

5) El tiempo propio de la acción comunicativa es el presente. La comunicación se da en unas coordenadas espacio-temporales específicas. No se da en el tiempo estándar, sino que la dimensión temporal propia de la comunicación es el tiempo interior. El tiempo propio de la acción comunicativa es el presente interior. Presente y simultaneidad aunque son términos muy relacionados entre sí, no deben ser confundidos. Presente indica el ahora. Simultaneidad, la confluencia de dos ahoras. Como todas las acciones, la acción de expresar y la de interpretar, se llevan a cabo siempre en presente. Sin embargo, en la comunicación, ambos presentes, el de la expresión y el de la interpretación, no tienen por qué coincidir con el tiempo estándar u objetivo. Puede darse comunicación cuando los copartícipes en la acción comunicativa, no comparten, ni el espacio físico ni el tiempo real.

Después de señalar estas características, podemos decir, que al hablar de comunicación nos referimos a algo concreto. La ciencia de la comunicación es la ciencia de esa realidad que acabamos de describir como una acción humana y social que consiste en compartir unos contenidos de conciencia que hacen referencia a un objeto real, que se da por sincronización de dos presentes distintos, en dos acciones también distintas: una expresiva y otra interpretativa.

En definitiva, el estudio científico de la comunicación es posible. Algunos autores, como Montes y Muñoz, afirman que: "La comunicación no puede convertirse en el centro de un modelo epistemológico solamente porque las demás ciencias: lógica, matemáticas, bioquímica, psicología, sociología, etc. hayan apor- 
tado a la teoría de la comunicación sus puntos de partida, sino porque la teoría de la comunicación está en disposición, o puede llegar a estarlo, de proporcionar, a las ciencias de las que se ha independizado, principios de revisión de sus mismos criterios de cambio y enriquecimiento epistemológico."

Este aspecto tiene una relevancia especial para el ámbito de la ciencia de la historia de la comunicación. En este ámbito, se ha producido un tratamiento científico del fenómeno comunicacional, a nivel social casi siempre. El utillaje metodológico empleado en estos estudios, ha estado más en relación con el peculiar momento que atravesaba la historiografía, que con las necesidades metodológicas propias y específicas de la ciencia de la comunicación. Me parece más acertado centrarse en el aprovechamiento de los resultados de la ciencia de la comunicación, en la medida en que las fuentes lo permitan, para conocer cuestiones del pasado que orienten en la búsqueda de soluciones, o principios de ellas, a problemas similares de la actualidad; sin descartar, por supuesto, el estudio de las estructuras y sistemas que organizaron la comunicación en las sociedades del pasado. En definitiva hay que tener en cuenta la perspectiva diacrónica en la ciencia de la comunicación.

Interesa ahora hacer algunas consideraciones sobre la historia, en concreto sobre los aspectos que mayor incidencia puedan tener en las tareas de un historiador de la comunicación.

Puede decirse que existe una crisis en los propios historiadores y en su producción historiográfica. En la medida en que todo es historiable, se produce como consecuencia la diversidad metodológica. Resulta casi tan difícil señalar un método histórico propio, como definir la ciencia de la comunicación. Es el resultado de la indefinición de la historia como disciplina científica.

Parece obligado abordar el tema central: el objeto de la historia. Hasta hace unos años esta expresión no constituía problema alguno pues el consenso acerca de su significado era general. La historia estudiaba las cosas hechas por los hombres, la trayectoria recorrida desde que hay memoria de ellos, las vicisitudes por las que fueron pasando a lo largo de los siglos, el modo como vivían y pensaban, su organización y las relaciones de unos con otros, todo ello constituía la materia sobre la que recaía la investigación. Una materia tan vasta y compleja, tan rica y variada, que no podía agotarse.

De entre los distintos elementos, integrantes de la realidad histórica, el pensamiento y las ideas, la política, las instituciones, la cultura, la sociedad, la economía, etc., los historiadores trataron unos con preferencia a otros, por las

3 MONTES, S. y MUÑOZ, A. "Un modelo comunicacional para el análisis antropológico" en Revista Española de Investigaciones Sociológicas, III, 1978, p. 111 
razones que fuera, pero abarcando cada vez más zonas. No es necesario subrayar que la historia política, las instituciones y la cultura han sido hasta ahora cultivadas con mayor atención que otros aspectos.

Después vino un sensible cambio en el campo de la historia o mejor aún, de los historiadores. Hace ya casi medio siglo comenzó a desprestigiarse la historia política, es decir la historia que se ocupaba de los hechos o acontecimientos, y en ese empeño, tanto la escuela de los Annales, como la de inspiración marxista y la de raíz sociológico-positivista coincidieron al menos en algunos puntos. De todos modos parece como si el resultado hubiese sido sustituir la historia política por la historia socio-económica. Los viejos historiadores como Ranke se habían despreocupado de la socioeconomía; los nuevos historiadores se despreocuparon de la historia política: en resumen, distintos exclusivismos.

En 1979 Lawrence Stone, en un rápido análisis del mundo de la historia, encontraba cuatro grupos de historiadores:

"Los viejos historiadores narrativos, principalmente historiadores políticos y biógrafos; los cliómetras que siguen actuando como traperos de la estadística; los historiadores sociales duros y sin sentimientos, todavía ocupados en analizar estructuras impersonales; y los historiadores de las mentalidades, que rastrean ideales, valores, utillajes mentales y pautas de comportamiento personal íntimo, cuanto más íntimo mejor." ${ }^{4}$

Los métodos históricos que criticaba Stone eran fundamentalmente tres: el marxista, el ecológico-demográfico de Annales y el cliométrico. Todos vinculados a esa idea de la historia como elemento sustancial. Los tres tuvieron su esplendor y decaimiento. Los tres fundaron su carácter científico en el método que utilizan. Las tres corrientes formaron destacados historiadores con aportaciones de referencia obligada. Por ello conviene estudiar sus aportaciones, especialmente por su relación e incidencia en la historia de la comunicación social. No me voy a detener ahora ni en la marxista, ni en la cliométrica. Sólo diré que una de las aportaciones más características del grupo de Annales es, la historia de las mentalidades.

En la conformación de las mentalidades contemporáneas, juegan un papel clave los medios de comunicación social. Por tanto, parece un buen anclaje metodológico para los historiadores de la comunicación. Es claro que la comunicación, y más con la aparición y proliferación de la comunicación de masas, conforma mentalidades, transmite valores y visiones del mundo, de la vida, de la sociedad y del hombre. En cualquier caso, el estudio de los medios y de los

4 STONE, L. "The revival of narrative: reflexions on a new old history" en Past and Present, $\mathrm{n}^{\circ}$ 85, 1979 
contenidos y la cuantificación de su penetración, pueden recibir una ayuda estimable de la historia de las mentalidades.

Señalaré a continuación algunas puntualizaciones sobre la historia y sus métodos y posibles puentes hacia la historia de la comunicación.

En primer lugar quiero indicar que la historia -entendida como ciencia o saber humanístico- es un ámbito de conocimientos a los que se llega por la investigación. La tarea de historiar comienza con la aproximación a los hechos y datos $^{5}$ y no concluye hasta la exposición de los resultados. Éstos, se presentan como explicación y reconstrucción, que completa, responde, interpreta y organiza, la realidad, desde una hipótesis de trabajo ${ }^{6}$. La historia supone una puesta en relación, un juego de relaciones ${ }^{7}$, un diálogo entre el presente y el pasado y un ejercicio de síntesis.

En segundo lugar, la comunicación es un actor protagonista de la historia. Los medios fabrican las ideas del lector con quien dialogan: forman gustos, canalizan reivindicaciones, crean mitos, acogen y cultivan ideologías, etc. La trascendencia de este papel, queda fuera de toda duda y es cada vez más importante. Por otra parte, la opinión pública la crean básicamente los medios de comunicación, por tanto, resulta necesario acudir a ellos para abordar el estudio de las mentalidades.

En tercer lugar, además de actores, los medios de comunicación son testigos del acontecer histórico: una fuente histórica rica y variada.

En cuanto al método y las técnicas historiográficas, que son modos de ordenar y valorar el material histórico, han variado según las circunstancias. Tanto lo que se considera significativo, como el enfoque para tratarlo, evoluciona con el tiempo del historiador. En ese sentido, cada generación hace su historia.

El problema de la historia actual es su necesidad de hacer síntesis coherentes. Para ello las aportaciones de los defensores de la teoría general de sistemas, han abierto esperanzas de carácter metodológico. Esta teoría trabaja sobre la similitud estructural que puede existir entre diversas realidades. Podemos definir sistema como un conjunto de elementos relacionados que tiene un fin común o como un conjunto de variables en funcionamiento ${ }^{8}$. Desde esta definición, cual-

5 SUÁREZ FERNÁNDEZ, Luis: «La exposición en el campo de la Historia. Nuevos temas y nuevas técnicas», en Once Ensayos sobre la Historia. Madrid 1976, p. 15

6 FEBVRE, L.: Combates por la Historia. Barcelona 1986

7 DUBY,G.: Diálogos sobre la Historia. Madrid 1988, p. 89.

8 La teoría general de sistemas se ofrece como un marco general que reclama el uso de otros métodos, a los que proporciona un contexto. El precedente de la teoría general de sistemas en los años 20 en Europa fue Von Bertalanffy. Esta teoría se ha ido imponiendo como un nuevo paradigma que superara el enfoque lineal tradicional causa-efecto. El fenómeno informativo puede caracterizarse como uno de los subsistemas sociales básicos de las civilizaciones contemporáneas, constituido a su vez por numerosos sistemas en interacción. Todo sistema es dinámico por definición, con un dinamismo que se apoya en una invariante, la estructura, que en su límite se presenta como variable. 
quier realidad podría ser considerada en función de los elementos que la componen. La teoría general de sistemas busca la similitud estructural entre realidades diversas para clasificarlas y ofrecer así una visión ordenada de la realidad que nos facilite su comprensión.

El problema, como siempre, es la realización concreta de esa tarea científica. La definición de sistema, exige un cierto grado de generalidad. La idea de considerarlos abiertos a influencias mutuas es muy positiva. Pero la dificultad persiste, porque exige una enorme capacidad de comprensión de sistemas de naturaleza muy diversa. Más aún, exige ponderación de los resultados de campos ajenos a su ámbito específico de investigación. En cualquier caso, es una línea de trabajo de gran utilidad para los historiadores de la comunicación, porque nuestro problema, se sitúa en unos límites más modestos: la compatibilización de sistemas informativos, con los sociales, culturales, económicos, políticos... de una época concreta. El objetivo se presenta pues alcanzable.

Primero porque entre la historia y la comunicación, existe una relación ininterrumpida que pasa a través del hombre. Este flujo tiene diversos niveles desde la teoría de los sistemas, ya que el sistema comunicativo es una estructura básica. Esta relación aporta datos significativos sobre la articulación social, el régimen político, el tipo de organización económica o el nivel e inquietudes culturales o ideológicas. Además, hay sincronía en el desarrollo de la estructura informativa y de la historia en general ${ }^{9}$. Un ejemplo claro se da en la Edad Contemporánea. La triple revolución demográfica, industrial y sociopolítica, impulsó el despegue de la prensa a una fase superior. La revolución demográfica generó una masa de lectores que sacó a la prensa de los reducidos límites en los que estaba sumida. La revolución industrial proporcionó la tecnología que permitió las grandes tiradas, el incremento de las ventas y los ingresos por publicidad. Así se genera el capital necesario para constituirse como una empresa. La revolución liberal, en lo político y en lo social, posibilitó la libertad de prensa y convirtió al periódico en un protagonista clave de la lucha revolucionaria. La influencia entre los sistemas es además interactiva. Por ejemplo, la prensa tuvo un papel importante en acontecimientos históricos.

El nacimiento de la historia de la comunicación está vinculado al desarrollo de la propia historia, sobre todo la contemporánea. En la medida en que los diversos aspectos de la comunicación tuvieron un protagonismo cada vez más claro, los historiadores de la política, de la literatura o de las ideas, iniciaron las investigaciones en este campo. De alguna manera, ese origen fue un buen comien-

9 GONZÁleZ, N. «Periodismo e Historia Contemporánea», en Estudios de Historia Moderna y Contemporánea. Homenaje a D.Jesús Pabón (II). Madrid. Revista de la Universidad Complutense, vol.XXVII, $\mathrm{n}^{\circ}$ 113,$1979 ;$ p. 374 
zo. Aseguró la conexión con los problemas de carácter general sin caer en especializaciones carentes de significado.

Schudson propuso profundizar en el método histórico propiamente dicho a partir de la pregunta "¿de qué modo influencian los cambios en la comunicación y cómo se ven influidos (los medios) por otros aspectos del cambio social? ${ }^{10}$. Se puede adoptar la pregunta como principio metodológico de partida para la investigación en la historia de la comunicación.

A mediados de la década de los setenta en España, dentro de las recién creadas facultades de Ciencias de la Información, se explicaba una materia denominada indistintamente «Historia del periodismo» o «Historia de la comunicación» cuyo programa se asentaba sobre dos raíces, la Historiografía y las Ciencias de la Información ya que los profesores que la impartían procedían de diferentes ramas de las ciencias sociales y humanísticas. El peso inicial de la Historiografía, fue, mucho más importante, tanto por su mayor tradición universitaria, con programas y textos de fácil acceso, como por la presencia magisterial de algunos catedráticos de Historia que, con visión de futuro, apoyaron y potenciaron la investigación y docencia en esa nueva especialidad. Tales ventajas tenían una cara negativa. La historiografía, en general, había pasado por alto la comunicación y sus medios. En el mejor de los casos, los historiadores habían utilizado los periódicos, nunca otros medios, como fuentes de segunda categoría o como instrumentos de referencia de otras fuerzas sociales, ideológicas o políticas, pero nunca como sector autóctono, como elemento esencial, en la explicación de las sociedades contemporáneas. Habían investigado, como señala Timoteo Álvarez ${ }^{11}$, fenómenos históricos tan importantes como el bolchevismo, el nazifascismo, el «new Deal», la guerra fría,... sin la más mínima referencia a los medios de comunicación, a los instrumentos de propaganda o a las técnicas de organización de la opinión pública. La historia del periodismo era, pues, una historia general, con los nombres y las situaciones de la historia política, ni siquiera de la "historia social", cuyos ciclos se llenaban con títulos de periódicos, para confirmar cómo los periodistas, aunque secundariamente, habían estado allí y habían sido testigos.

Este modo de hacer requería un cambio. Desde el momento en que las masas se constituyeron en la fuerza social dominante, hace más de cien años, la comunicación y sus medios operaron como uno de los elementos esenciales de evolución y organización social. Incluso han sido con mucha frecuencia elementos definitivos, que han dado personalidad, estructura y sentido, a situaciones históri-

10 SCHUDSON, M. "Enfoques históricos de la comunicación” en JENSEN, K. B. y JAWKOWSKI, N. W. (ed.) Metodologías cualitativas de la investigación en comunicación de masas. Bosch. Barcelona, 1993, 211-228

11 Vid. ÁlVAREZ, J. T. Historia de los medios de comunicación en España. Ed. Ariel. Barcelona. 1989, p. 8 
cas recientes: las ya citadas, por ejemplo, de la guerra fría, el nazifascismo. etc. En los años 80 Timoteo Alvarez y otros, propusieron una "idea rupturista" como él mismo dice, frente a la Historiografía tradicional; han puesto en pie métodos, trabajos, ejemplos, que manifiestan la función, la identidad y la fuerza de la comunicación en las sociedades, sobre todo, contemporáneas ${ }^{12}$.

Visto esto, la historia de la comunicación social, ha de contemplarse como un sistema internamente lógico. Incluye subsistemas que se encuentran interrelacionados. La interdependencia, presenta diversos grados que se pueden estudiar de manera sincrónica o diacrónica.

La coordenada sincrónica integra varios subsistemas. Uno es el legal es decir, las relaciones entre el poder y la comunicación. Otro el estructural que comprende el régimen, la administración y el organigrama de las empresas. Además existe el subsitema que actúa de infraestructura con la variable tecnológica y la social. En esta misma coordenada sincrónica, también hay que tratar de los protagonistas de la organización informativa que en cada momento ejercen la dirección de los medios.

La coordenada diacrónica, o temporal, exige considerar los diversos tipos de duración.

La historia de la comunicación social se ha solido dividir según dos criterios. Uno la considera con autonomía propia y en consecuencia traza divisiones por los hitos informativos claves. En España un planteamiento riguroso de periodización de la historia desde una perspectiva comunicacional ha sido el desarrollado por el profesor Timoteo Alvarez. Se inspira en los tres tipos de ciclos que para el análisis histórico acuñó Braudel: el estructural o de tiempo largo, el coyuntural o de tiempo medio y el de eventos o de tiempo corto.

El segundo modo de periodizar la historia de la comunicación social es hacer coincidir las etapas de la evolución de la comunicación, con las grandes y pequeñas edades y períodos del devenir histórico ${ }^{13}$. Es la opción de los manuales más conocidos de historia del periodismo, que siguen la división clásica en edades. A pesar de las dificultades que implica y de la inestabilidad de las fronteras entre las diversas etapas, mantiene su utilidad.

En cualquier caso -hoy por hoy- el intento de Timoteo Álvarez parece una síntesis práctica y eficaz. Así lo demuestra, por otra parte, el hecho de que partiendo de bases diferentes a la historia tradicional, haya venido a confirmar ésta, en líneas generales. De todas formas él propone una cierta revisión de las fronteras temporales. Sugiere que se tengan en cuenta algunos de los momentos de

12 ÁLVAREZ, J. T. (cit. pp. 7 y 8)

13 Este es el caso, por ejemplo, de la síntesis citada de ALBERT y TERROU y de la mayor parte de los manuales y monografías de historia de la prensa mencionadas en notas anteriores. 
ruptura más importantes de la historia del periodismo. Estos son: la invención de la imprenta -verdadero agente del despegue del sector de la comunicación moderna, la aparición de la prensa periódica en el siglo XVII, y los inicios de una prensa para las masas en el siglo XIX.

Para los alumnos que estudian publicidad y relaciones públicas, periodismo y comunicación audiovisual, y que se preparan en principio para una profesión volcada más hacia el activismo y el momento que hacia la reflexión y el pasado, puede resultar conveniente conocer el origen, también histórico de la terminología y de los conceptos de una profesión con siglos de praxis y apenas décadas de sistematización y teoría.

Al estudiar o investigar sobre historia de la comunicación, no se trata de ofrecer el rastreo, catalogación, ordenamiento y tratamiento de los periódicos, alabando sus méritos e intentando conocer los mecanismos de su funcionamiento. Esa es la fase primigenia de cualquier desarrollo científico, la de localización y crítica de sus fuentes y material de trabajo, que por razones específicas del sector de la información, no comenzó a ser superada hasta finales de los setenta. Lo que se pretende es entre otras cosas, un conocimiento de la historia, sobre todo de la edad contemporánea, a partir de algo esencial, especialmente para el siglo XX, que es la información. Parece necesaria una revisión de la edad contemporánea desde una perspectiva que incluya como valor fijo la variable información / comunicación / propaganda.

Lo que importa es encontrar modelos, sistemas, fórmulas de éxito y fracaso, razones que potencien la voluntad de riesgo y que demuestren el valor social y personal de la comunicación. De forma pasiva o activa todos estamos ante los medios de comunicación. Nuestra existencia está condicionada y articulada por ellos. Conocer su trayectoria o evolución, tiene por eso un grandísimo interés, que podemos aplicar con utilidad al presente y al futuro.

El conocimiento y profundización en la historia de la comunicación nos permite someter las informaciones que nos llegan a un análisis lúcido, para no quedar atrapados en las redes de las ideologías. En definitiva, ayuda a entender la complejidad de la realidad y a comprender, por la experiencia de sociedades anteriores, cómo actúan los diversos elementos culturales y sociales. 